\title{
Duodenum/Ampulla/Jejunum/lleum Neuroendocrine Tumor pT2 TNM Finding v7
}

National Cancer Institute

\section{Source}

National Cancer Institute. Duodenum/Ampulla/Jejunum/lleum Neuroendocrine Tumor

pT2 TNM Finding v7. NCI Thesaurus. Code C90086.

Duodenum/ampulla/jejunum/ileum neuroendocrine tumor invading muscularis propria or is more than $1 \mathrm{~cm}$ in size (small intestinal tumors); for ampullary tumors: tumor more than $1 \mathrm{~cm}$. (from AJCC 7th Ed.) 